\title{
Dynamic response of double-beam system with nonlinear viscoelastic layer to moving load
}

\author{
Piotr Koziol ${ }^{1, *}$ and Rafat Pilecki ${ }^{2}$ \\ ${ }^{1}$ Cracow University of Technology, Faculty of Civil Engineering, ul. Warszawska 24, 31-155 Kraków, \\ Poland \\ ${ }^{2}$ Former student of the Cracow University of Technology, ul. Warszawska 24, 31-155 Kraków, Poland
}

\begin{abstract}
In previous papers, the problem of double-beam system resting on viscoelastic foundation was solved with the assumption of nonlinear foundation stiffness. This multilayer model finds application in railway modelling, where rails are represented by the infinite Euler-Bernoulli beams and sleepers are modelled as a rigid body. In this paper, another assumption is made. The layer connecting two Euler-Bernoulli beams has nonlinear stiffness. This assumption is related to laboratory tests of fastening systems. These tests show that the stiffness of fasteners and rail pads is nonlinear and this factor should be taken into account in detailed analysis of dynamic features. Therefore inclusion of nonlinearity in double-beam system is justified. The physical model presented in this paper consists of two infinitely long beams connected by viscoelastic layer with nonlinear stiffness and resting on viscoelastic foundation. The mathematical model is described by two coupled fourth order partial differential equations of motion with homogeneous boundary conditions. The system is solved by using the Fourier transform and Adomian's decomposition, combined with the wavelet based approximation of the response using Coiflet filters. The error index for Adomian series is proposed and the approximate solution for vertical vibrations is shown along with computational examples for some systems of parameters.
\end{abstract}

\section{Introduction}

The problem of double-beam dynamics is very important for engineering. One of the most crucial fields using the idea of such a system is modelling of beam-foundation structures subjected to moving loads. Continuously improving transportation systems and the need of fulfilling structural conditions necessary for making structures more safe and convenient for passengers led to the necessity of prediction of their dynamic behaviour. In the literature, one can find different models representing rail tracks on rigid foundation [1, 2]. One should underline that these models are usually solved in linear case, whereas nonlinear features are discussed in case of numerical approaches. Analytical solutions of nonlinear systems are relatively rare, although highly valued due to their universality and usefulness in parametrical studies.

\footnotetext{
* Corresponding author: pkoziol@pk.edu.pl
} 
The model presented in this paper is based on the double-beam system described and solved previously [3]. The assumption of viscoelastic foundation supporting structure with nonlinear description of its stiffness was introduced in past papers. The analytically solved and parametrically analysed system was directly implemented in the analysis of multilayer rail track model, where the rail was represented by the Euler-Bernoulli beam (upper beam) and the sleepers were described as a rigid body (lower beam with zero damping) $[4,5]$.

Some experimental studies showed that the layer between two beams (e.g. fastening system for rail track) should be also considered as a nonlinear one. In the literature, one can observe a lack of analytical solutions of such systems. Some methods can be found for mechatronics applications, where the beams are considered as finitely long, whereas the case of infinitely long system remains unsolved.

Linear system is relatively easy to solve. In reality, nonlinearities should not be omitted [6-8], which render this problem more difficult. The system presented in this paper consists of two infinitely long Euler-Bernoulli beams connected by the viscoelastic layer with included nonlinearity of stiffness in its classical cubic form. This structure rests on viscoelastic foundation and it is subjected to moving set of forces harmonically varying in time.

\section{Mathematical model}

The vertical vibrations of the double-beam system (with nonlinear stiffness of the lower layer) is governed by the system of two coupled nonlinear fourth order partial differential equations:

$$
\begin{gathered}
E I_{1} \frac{\partial^{4} u}{\partial x^{4}}+m_{1} \frac{\partial^{2} u}{\partial t^{2}}+c_{1}\left(\frac{\partial u}{\partial t}-\frac{\partial w}{\partial t}\right)+k_{1}(u-w)+k_{N} u^{3}=P(x, t) \exp (i \Omega t) \\
E I_{2} \frac{\partial^{4} w}{\partial x^{4}}+m_{2} \frac{\partial^{2} w}{\partial t^{2}}+c_{2} \frac{\partial w}{\partial t}+k_{2} w-c_{1}\left(\frac{\partial u}{\partial t}-\frac{\partial w}{\partial t}\right)-k_{1}(u-w)-k_{N} u^{3}=0
\end{gathered}
$$

where $u$ and $w$ are the vertical displacements of upper and lower beam, respectively.

Other parameters denote: $E I_{1}, m_{1}$ - bending stiffness and mass of upper beam; $E I_{2}, m_{2}$ - bending stiffness and mass of lower beam; $c_{1}, k_{1}$ and $c_{2}, k_{2}$ - viscous damping and linear part of stiffness of the layer between beams and the foundation, respectively. The nonlinear part of stiffness introduced in the connecting layer is represented by monomial $u^{3}$ with parameter $k_{N}$ [8]. Homogeneous boundary conditions are applied in order to suppress vibrations of beams in the infinity (at points far enough from the point of load, the central line deflection, its slope and curvature tend to zero): in reality, it is enough to do it on relatively big distance from the source of vibrations (moving forces). The force is harmonically varying in time with frequency $\Omega=2 \pi \cdot f_{\Omega}$.

The load acting on upper beam, considered in this paper, consists of two identical forces moving along the beam with constant speed $V$ at constant distance $l$ between them. Each force is described in space-time domain by the following formula $[5,8]$ :

$$
P(x, t)=\frac{P}{r} \cos ^{2}\left(\frac{\pi(x-V t)}{2 r}\right) H\left(r^{2}-(x-V t)^{2}\right)
$$

where $H($.$) is the Heaviside step function and 2 r$ is the span of single force.

\section{Solution}

The system of equations (1)-(2) can be uncoupled and solved by applying the Fourier transform. In order to make possible using the Fourier transform, the developed before 
technique based on Adomian's decomposition is applied [9]. It assumes that the solution of the system can be represented by series of functions, where the first term is associated with linear problem and other terms must be determined by using so called Adomian polynomials [9-11]. Because of relatively high complexity of formulas arising from this approach, the wavelet based approximation is used for re-transformation of the solution obtained in the transform domain. In previous papers, it was shown that the coiflet based approximation combined with Adomian's expansion leads to results that can be effectively used in parametrical analysis.

In order to keep convergence of the obtained series representing the dynamic response of the system, one should follow the rules formulated for other systems, e.g. [8, 11]. These are: stabilization condition with regard to physical parameters of structure and, for appropriate choice of the approximation order, controlling of so called "error index" defined by the following formulas, see also [12]:

$$
0 \leq \frac{\left\|u_{j+1}\right\|}{\left\|u_{j}\right\|}<1
$$

and

$$
u_{n}^{e r}=\frac{\left|\left\|S_{n}^{u}\right\|-\left\|S_{n-1}^{u}\right\|\right|}{\left\|S_{n}^{u}\right\|}
$$

for the terms of Adomian series $\left(j=0,1,2, \ldots\right.$ and $\left\|u_{j}\right\| \neq 0$ ) of both beams response ( $u$ and w) with the norm

$$
\left\|u_{j}\right\|=\max _{x}\left|\operatorname{Re}\left[u_{j}(x)\right]\right|
$$

where $S_{n}^{u}$ denotes the $n$-th partial sum of the approximate solution obtained in physical domain. Condition (4) allows to obtain solution with assumed level of accuracy.

For simplicity of calculations, a moving coordinate system can be easily introduced: $\tilde{x}=x-V t$.

\section{Examples and discussion}

The system has been satisfactorily solved, i.e. the obtained solution can be effectively used in parametrical analysis. In order to show efficiency of the developed semi-analytical approach, some numerical examples are presented. The system of parameters similar to this one used in past papers is assumed [3]:

$P=5 \cdot 10^{5} \mathrm{~N} / \mathrm{m}, k_{N}=4 \cdot 10^{13} \mathrm{~N} / \mathrm{m}^{4}, E I_{1}=10^{7} \mathrm{Nm}^{2}, m_{1}=100 \mathrm{~kg} / \mathrm{m}, k_{1}=4 \cdot 10^{7} \mathrm{~N} /$ $\mathrm{m}^{2}, \quad c_{1}=0.06 \cdot \sqrt{k_{1} \cdot m_{1}}, E I_{2}=1.5 \cdot 10^{9} \mathrm{Nm}^{2}, \quad m_{2}=3500 \mathrm{~kg} / \mathrm{m}, \quad k_{2}=5 \cdot 10^{7} \mathrm{~N} / \mathrm{m}^{2}$, $c_{2}=0.06 \cdot \sqrt{k_{2} \cdot m_{2}}$.

Figure 1 shows examples of the system response represented by the real part of nonlinear solution obtained for vertical vibrations of beams. One can observe that the amplitude of lower beam vibrations is smaller than in the case of the upper beam. The amplitudes are comparable for two considered lower and higher frequencies.

Figure 2 presents comparison of linear and nonlinear solutions for different velocities of loads and different distances between separated forces. The upper beam is much more sensitive for nonlinear factor included in the model, whereas the lower beam reflects almost the same behaviour in linear and nonlinear cases. In such situations, when the change of parameters leads to only marginal changes in model characteristics, it is convenient to use 
the complex modulus of solution, which represents so called "maximal response" and allows to analyse the system sensitivity in more detail. Some comparative examples of the maximal response obtained by using the complex modulus are shown in figure 3 . Figures $3 \mathrm{e}$ and $3 \mathrm{f}$ shows zoomed fragments of $3 \mathrm{c}$ and $3 \mathrm{~d}$, respectively.

(a)

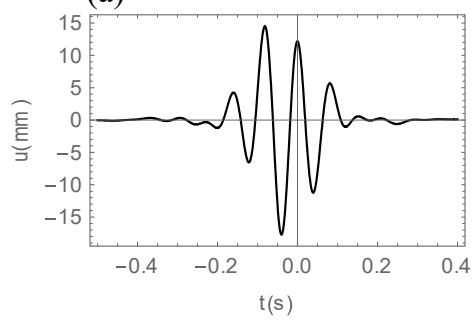

(c)

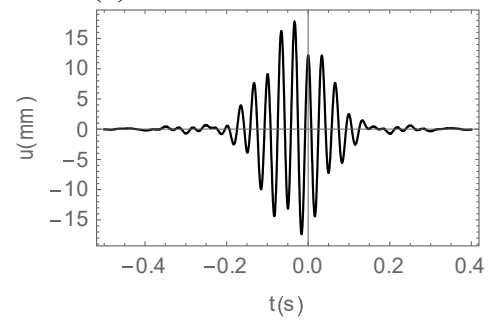

(b)

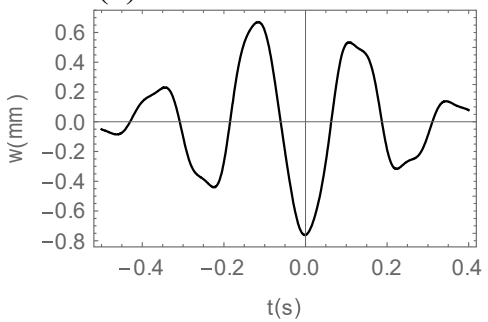

(d)

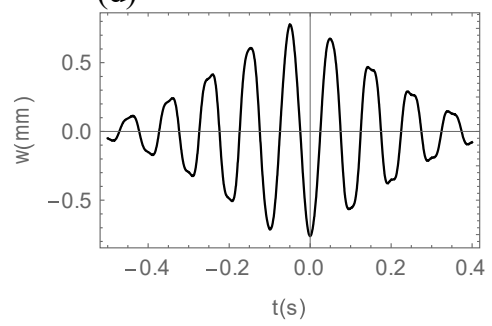

Figure 1. Vertical vibrations (nonlinear solution) of upper (a)-(c) and lower (b)-(d) beams: (a)-(b) $V=15 \mathrm{~m} / \mathrm{s}, l=1 \mathrm{~m}, f_{\Omega}=4 \mathrm{~Hz}$; (c)-(d) $V=15 \mathrm{~m} / \mathrm{s}, l=1 \mathrm{~m}, f_{\Omega}=10 \mathrm{~Hz}$.

(a)

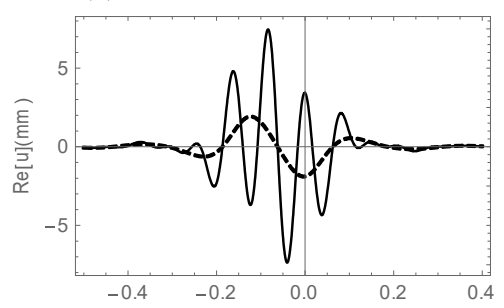

(c)

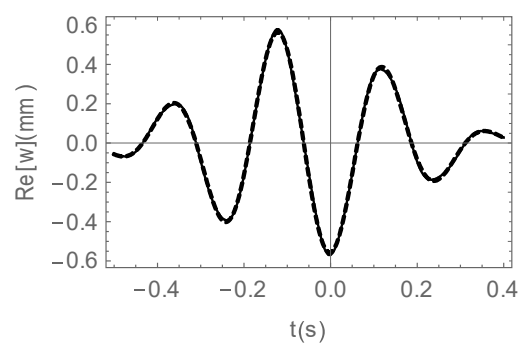

(b)

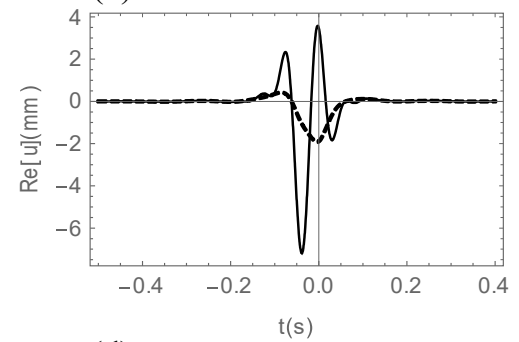

(d)

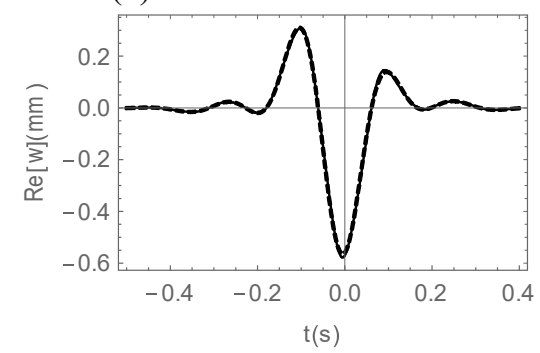

Figure 2. Vibrations of upper beam (a)-(b) and lower beam (c)-(d): (a) and (c) $V=15 \mathrm{~m} / \mathrm{s}, l=2 \mathrm{~m}$, $f_{\Omega}=4 \mathrm{~Hz}$; (b) and (d) $V=40 \mathrm{~m} / \mathrm{s}, l=2 \mathrm{~m}, f_{\Omega}=4 \mathrm{~Hz}$ (linear - dashed, nonlinear - solid). 
(a)

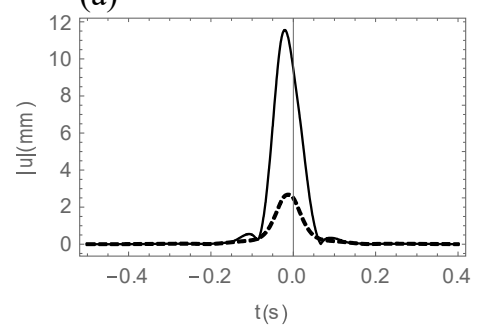

(c)

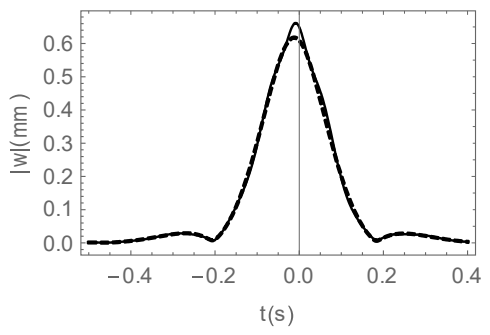

(e)

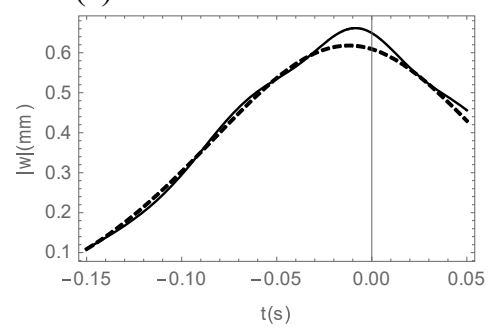

(b)

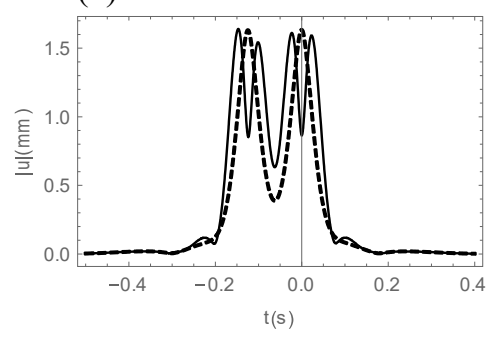

(d)

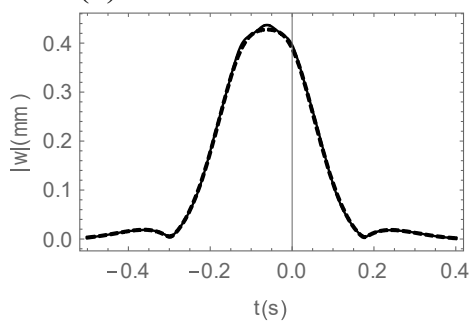

(f)

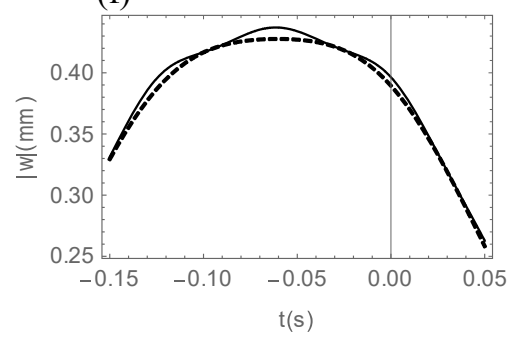

Figure 3. The maximal response of upper beam (a)-(b) and lower beam (c)-(f): (a), (c) and (e) $V=$ $40 \mathrm{~m} / \mathrm{s}, l=1 \mathrm{~m}, f_{\Omega}=4 \mathrm{~Hz}$; (b), (d) and (f) $V=40 \mathrm{~m} / \mathrm{s}, l=5 \mathrm{~m}, f_{\Omega}=4 \mathrm{~Hz}$ (linear - dashed, nonlinear - solid).

The presented examples show that parametrical analysis of the investigated system is possible, however detailed study and synthetic analysis are left for further work.

\section{Conclusions}

The double-beam system with nonlinear stiffness of layer connecting two infinitely long Euler-Bernoulli beams is solved by using a hybrid method based on semi-analytical approximations. The obtained solution allows parametrical analysis of the model with possibility of controlling of accepted accuracy level. Numerical examples show that the assumed nonlinear factor strongly influences the system for some systems of parameters. The influence of nonlinearity becomes stronger when the distance between forces decreases. Further analysis of the system is needed before its application to real mechanical systems investigation, in order to recognize all important features of the developed technique. 


\section{References}

[1] L. Fryba, Thomas Telford Ltd., London, (1999)

[2] M.F.M. Hussein, H.E.M. Hunt, JSV, 297, 37-54, (2006)

[3] P. Koziol, J. Theor. Appl. Mech., 52, 3, 687-697 (2014)

[4] P. Koziol, Proceedings of ICoEV2015, International Conference on Engineering Vibration, Ljubljana, Slovenia, 7-10 September 2015, paper 273, (2015)

[5] P. Koziol, Proceedings of the Fifteenth International Conference on Civil, Structural and Environmental Engineering Computing, Civil-Comp Press, Stirlingshire, UK, Paper 199 (2015)

[6] T. Dahlberg, Proceedings of Fifth International Conference on Structural Dynamics, Munich (2002)

[7] M.H. Kargarnovin, D. Younesian, D.J. Thompson, C.J.C. Jones, Comput. Struct., 83, 1865-1877 (2005)

[8] P. Koziol, Mech. Syst. Signal Process., 79, 174-181, (2016)

[9] G. Adomian, Kluwer Academic Publishers, Dordrecht, (1989)

[10] G. Adomian, Kluwer, Boston, MA, (1994)

[11] P. Koziol, VDM Verlag Dr. Müller, Saarbrucken, (2010)

[12] Z. Hryniewicz, P. Koziol, Proceedings of the First International Conference on Railway Technology: Research, Development and Maintenance, Civil-Comp Press, Stirlingshire, UK, Paper 112 (2012) 